\title{
Analysis of cuprous oxide-based ultra-compact nanoplasmonic coupler
}

\author{
Md. Ghulam Saber $\cdot$ Rakibul Hasan Sagor
}

Received: 22 February 2014/ Accepted: 26 March 2014/Published online: 11 April 2014

(C) The Author(s) 2014. This article is published with open access at Springerlink.com

\begin{abstract}
A cuprous oxide-based ultra-compact nanoplasmonic coupler has been proposed and analyzed numerically. Numerical simulations show that a coupling efficiency of $56 \%$ has been achieved at the optical communication wavelength. The proposed nanoplasmonic coupler has the advantage of operating at broad frequency range. The fabrication process of the coupler is also easier compared to other proposed structures since it is a flat rectangular-shaped coupler and does not require any tapering at any point.
\end{abstract}

Keywords Surface plasmon polariton - Plasmonic waveguide $\cdot$ Nanoplasmonic coupler $\cdot$ Finite-difference time domain

\section{Introduction}

Plasmonic waveguides have been a field of extensive research interest for the past few years. In particular, the metal-insulator-metal (MIM) configuration of the plasmonic waveguide has the ability to squeeze the optical modes within the insulator layer on deep sub-wavelength scales (Barnes et al. 2003; Zia et al. 2004). This amazing property of the plasmonic waveguides has a promising role in minimizing the size of the integrated photonic devices and acts as a bridge between conventional optics and sub-wavelength electronics devices. The confinement of the optical mode depends on the thickness of the

Md. G. Saber $(\bowtie) \cdot$ R. H. Sagor

Department of Electrical and Electronic Engineering, Islamic

University of Technology, Board Bazar, Gazipur 1704,

Bangladesh

e-mail: gsaber@iut-dhaka.edu insulator layer. The thinner the layer, the more confined is the optical mode. However, the tradeoff is increased propagation power loss in the metallic layer which limits the distance of how much the surface-plasmon-polariton (SPP) mode can propagate. Even the fabrication-related disorders have far less impact on the propagation loss than the losses that occur in metallic layers. This problem can be addressed using both dielectric and plasmonic waveguide in the same chip. The dielectric waveguide will carry the optical mode while the plasmonic waveguide will address the sub-wavelength scale issue. This calls for the need of efficient coupling of optical modes from the dielectric waveguide to the plasmonic waveguide. Therefore, designing efficient nanoplasmonic couplers with different materials and structures can be a pioneering step in miniaturization of the integrated photonic devices.

In the past years, several plasmonic couplers have been proposed by different researchers. Veronis and Fan (2007) proposed a coupler with multi-section tapers. Ginzburg and Orenstein (2007) reported a $\lambda / 4$ coupler to couple optical modes from a $0.5-\mu \mathrm{m}$ - to 50 -nm-wide plasmonic waveguide. Pile and Gramotnev (2006) presented an adiabatic and a non-adiabatic tapered plasmonic coupler. Wahsheh et al. (2012) reported an analysis on nanoplasmonic air-slot coupler and its fabrication steps.

In this paper, we present a novel design and analysis of a nanoplasmonic coupler using cuprous oxide, based on the finite-difference time-domain method (Yee 1966). To the best of our knowledge, this is for the first time one proposes and analyzes a nanoplasmonic coupler using cuprous oxide. We have achieved a coupling efficiency of $56 \%$ at the telecommunication wavelength. The advantage of this design is that it can operate at a wide range of frequencies and is easier to fabricate since it is a simple flat terminal 
waveguide without any tapering placed at the entry of the MIM plasmonic waveguide.

\section{Formulation of the materials and the structure}

Material models

The frequency-dependent permittivity function of singlepole pair Lorentz model is given by (Kunz and Luebbers 1993),

$\varepsilon_{\mathrm{r}}(\omega)=\varepsilon_{\infty}+\frac{\omega_{\mathrm{o}}^{2}\left(\varepsilon_{\mathrm{s}}-\varepsilon_{\infty}\right)}{\omega_{\mathrm{o}}^{2}+j 2 \delta \omega-\omega^{2}}$

where, $\varepsilon_{\infty}$ is the infinite frequency relative permittivity, $\varepsilon_{\mathrm{s}}$ is the zero frequency relative permittivity, $\boldsymbol{j}$ is the imaginary unit, $\delta$ is the damping coefficient and $\omega_{o}$ is the frequency of the pole pair.

The frequency-dependent permittivity function of Lorentz-Drude 6 (six)-pole model is given by (Kunz and Luebbers 1993),

$\varepsilon_{\mathrm{r}}(\omega)=1-\frac{f_{\mathrm{o}} \omega_{\mathrm{p}}^{2}}{\omega^{2}-j \Gamma_{\mathrm{o}} \omega}+\sum_{i=1}^{5} \frac{f_{\mathrm{i}} \omega_{\mathrm{p}}^{2}}{\omega_{\mathrm{oi}}^{2}+j \Gamma_{\mathrm{i}} \omega-\omega^{2}}$

where, $\omega_{\mathrm{p}}$ is the plasma frequency, $\Gamma_{\mathrm{i}}$ is the damping frequency, $f_{\mathrm{i}}$ is the oscillator strength, $\boldsymbol{j}$ is the imaginary unit and $\omega_{\mathrm{oi}}$ is the resonant frequency.

We have used single-pole Lorentz model to account for the dispersive property of cuprous oxide $\left(\mathrm{Cu}_{2} \mathrm{O}\right)$ and six-pole Lorentz-Drude model to integrate the dispersion property of silver (Ag) in the simulation model. An excellent agreement has been achieved with the experimental values (Palik 1998) by fitting $\mathrm{Cu}_{2} \mathrm{O}$ to the singlepole with $\varepsilon_{\infty}=(1.41)^{2}, \quad \varepsilon_{\mathrm{s}}=(2.49)^{2}, \quad \delta=6.1 \times 10^{10}$ $\mathrm{rad} / \mathrm{s}$ and $\omega_{\mathrm{o}}=0.53 \times 10^{16} \mathrm{rad} / \mathrm{s}$. The modeling parameters for $\mathrm{Ag}$ have been determined by Rakic et al. (1998).

\section{Structure formulation}

We have developed the 2D simulator based on the finitedifference time-domain (FDTD) method proposed by Yee (1966). A general auxiliary differential equation (ADE)FDTD algorithm is used to integrate the frequencydependent dispersion properties of the materials (Alsunaidi and Al-Jabr 2009; Al-Jabr and Alsunaidi 2009). This algorithm is useful where materials with different dispersion properties are present. The perfectly matched layer (PML) has been used to avoid reflection of incident wave from the boundaries (Berenger 1994).

Considering the material dispersion, the frequencydependent electric flux density can be given as

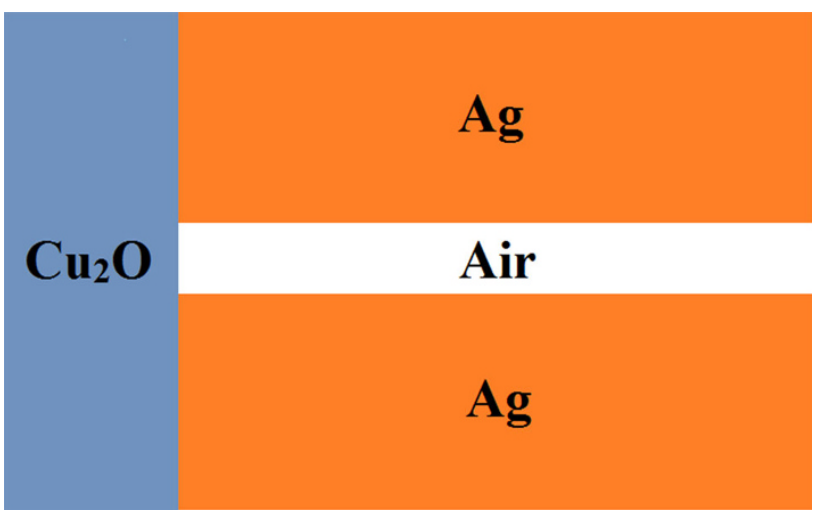

Fig. 1 Schematic diagram of the coupling structure used for the numerical analysis

$D(\omega)=\varepsilon_{0} \varepsilon_{\infty} E(\omega)+P(\omega)$.

The general Lorentz model is given by

$P(\omega)=\frac{a}{b+j c \omega-d \omega^{2}} E(\omega)$

which can be written in time domain through inverse Fourier transform as

$b P(t)+c P^{\prime}(t)+d P^{\prime \prime}(t)=a E(t)$.

The FDTD solution for the first-order polarization of Eq. (3) can be expressed as

$P^{n+1}=C_{1} P^{n}+C_{2} P^{n-1}+C_{3} E^{n}$

where

$C_{1}=\frac{4 d-2 b \Delta t^{2}}{2 d+c \Delta t}, \quad C_{2}=\frac{-2 d-c \Delta t}{2 d+c \Delta t}, \quad C_{3}=\frac{2 a \Delta t^{2}}{2 d+c \Delta t}$.

The values of $C_{1}, C_{2}$ and $C_{3}$ depend on the material under consideration.

Finally the electric field intensity becomes

$E^{n+1}=\frac{D^{n+1}-\sum_{i}^{N} P^{n+1}}{\varepsilon_{0} \varepsilon_{\infty}}$

where $D^{n+1}$ is the update value of the electric flux density calculated using the FDTD algorithm.

\section{Structure specifications and simulation}

The proposed nanoplasmonic coupler structure that has been used for simulation is given in Fig. 1. Here, the width of the $\mathrm{Cu}_{2} \mathrm{O}$ layer has been taken as $300 \mathrm{~nm}$ and the width of the air layer in the MIM waveguide has been taken as $60 \mathrm{~nm}$.

A monochromatic point source has been used to excite the optical modes. Since the coupler can operate at a broad frequency range, we have used input signals of 


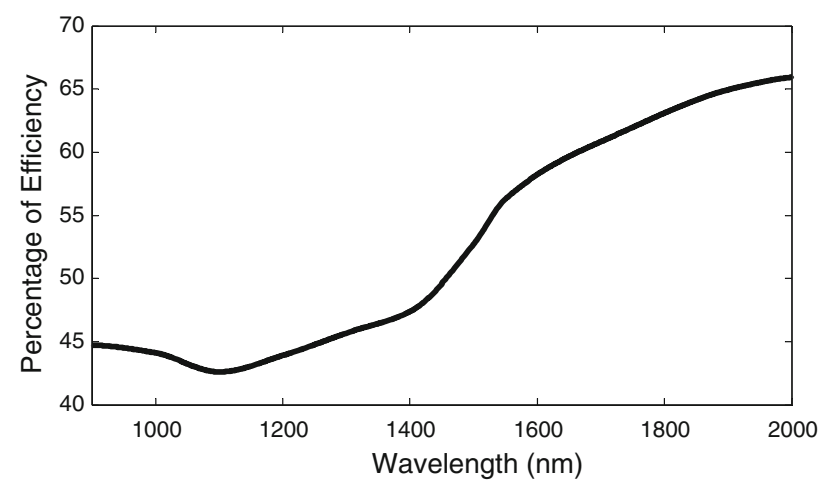

Fig. 2 Coupling efficiency of the proposed nanoplasmonic coupler

wavelengths ranging from 900 to $2,000 \mathrm{~nm}$. The reason we have limited our simulation within this wavelength range is that the modeling parameters for the materials we have used are applicable within this wavelength boundary only.

To get accurate results and maintain the courant stability criteria (Taflove and Hagness 2000), we have taken $\Delta x=5 \mathrm{~nm}, \Delta y=5 \mathrm{~nm}$ and the time step as $\Delta t=\frac{0.95}{c \sqrt{\frac{1}{\Delta x^{2}}+\frac{1}{\Delta y^{2}}}}$.

We have defined the coupling efficiency as the ratio of the transmitted power into the MIM waveguide to the incident power in the input dielectric waveguide. The incident power of the fundamental mode has been measured right before the interface between dielectric and MIM waveguide and the transmitted power has been measured right after the interface.

The reflection coefficient, return loss and voltage standing wave ratio (VSWR) have also been determined to analyze the performance of the coupler. The method we have used for calculating reflection coefficient is as follows. First, an optical mode has been incident in the dielectric waveguide when there is no plasmonic waveguide. The value of the electric field is then recorded at one point. This represents the value of the incident wave. Then, the same thing has been done with the plasmonic waveguide. This time the electric field represents the value of the incident wave plus the reflected wave since some part of the incident wave will be reflected by the MIM waveguide due to the difference in dispersion property of the materials. Therefore, we can calculate the reflected wave by subtracting the incident wave from this value. The reflection coefficient is then calculated by taking the maximum of the ratio of the reflected wave to the incident wave. This has been done for all the input signal wavelengths for which we have run the simulation. After determining the reflection coefficient, we have determined the return loss and VSWR using analytical formulas.

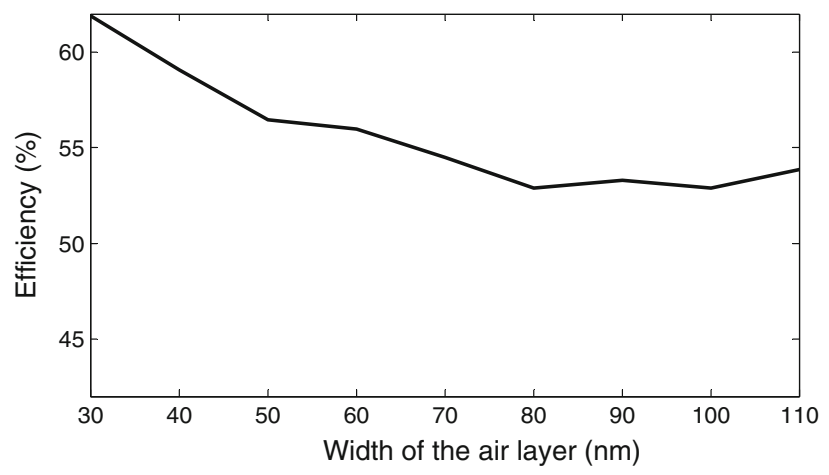

Fig. 3 Variation of coupling efficiency with varying width of the air layer

(i)
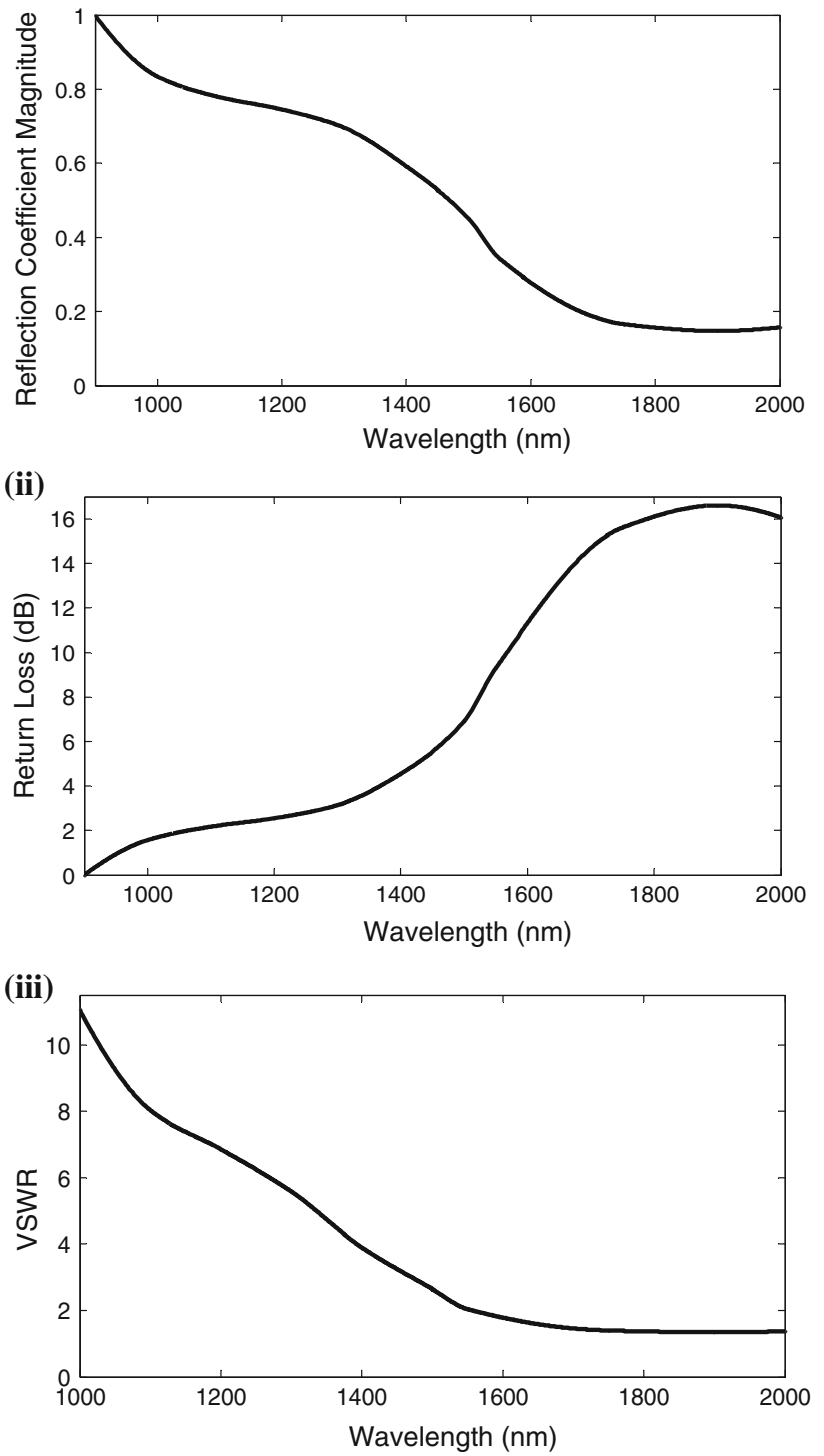

Fig. 4 Numerically calculated i reflection coefficient, ii return loss, iii voltage standing wave ratio (VSWR) of the proposed nanoplasmonic coupler 


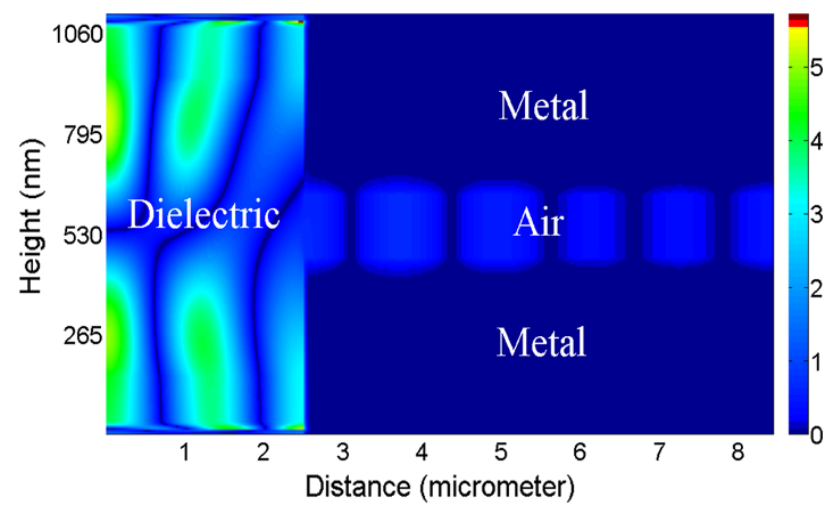

Fig. 5 Electric field distribution in the proposed nanoplasmonic coupler

\section{Results and discussion}

The coupling efficiency of the proposed nanoplasmonic coupler using cuprous oxide has been determined for different input signal wavelengths and presented in Fig. 2. From the figure, it can be observed that the coupling efficiency keeps on increasing as we increase the input signal wavelength reaching about $66 \%$ at the input signal wavelength of $2,000 \mathrm{~nm}$. The optical communication wavelength is of particular interest for us and at this wavelength the coupling efficiency is $56 \%$.

The variation of the coupling efficiency as a function of the width of the air layer has been depicted in Fig. 3. We have varied the width from 30 to $110 \mathrm{~nm}$ in the step of $10 \mathrm{~nm}$. In each case, the wavelength of the input signal has been kept fixed at $1,550 \mathrm{~nm}$. A maximum efficiency of $61.89 \%$ has been achieved for $30 \mathrm{~nm}$ width and a minimum of $52.9 \%$ has been observed when the width is $100 \mathrm{~nm}$. However, for the ease of fabrication we have kept the width at $60 \mathrm{~nm}$ while doing the final simulation.

The numerically calculated reflection coefficient has been given in Fig. 4i. From the figure, it can be observed that the reflection coefficient is decreased as the input signal wavelength is increased and at the optical communication wavelength the reflection coefficient is 0.34 which indicates that the amount of reflection is very small for the proposed coupler.

From the numerically calculated reflection coefficient, we have determined the return loss and the voltage standing wave ratio which are given in Fig. 4ii, iii. The return loss is increasing with the increase in input signal wavelength and it is $9.32 \mathrm{~dB}$ at the optical communication wavelength which indicates that the impedance mismatch is low at this wavelength. The VSWR is also very low at this wavelength having a value of 2.04 only.

The electric field distribution inside the coupling structure for cuprous oxide has been presented in Fig. 5. From the colormap of the figure, it is understood that the field intensity is higher in the dielectric waveguide than the plasmonic waveguide. Besides, due to higher losses in the metallic layer the field intensity decays if one goes into the top and bottom metallic layers from the dielectric region of the plasmonic waveguide.

The coupler proposed by Wahsheh et al. (2012) provides a theoretical efficiency of $50 \%$ at the optical communication wavelength whereas our proposed coupler provides a theoretical efficiency of $56 \%$ at the same input signal wavelength. The proposed couplers of Veronis and Fan (2007) and Pile and Gramotnev (2006) contain multisection tapers which are difficult to fabricate at the nanoscale. But our proposed structure is easier to fabricate since we have not used any tapered interface. Therefore, it is evident that our proposed coupler using cuprous oxide provides noticeably better performance in terms of efficiency and ease of fabrication.

\section{Conclusion}

We report the design and analysis of a nanoplasmonic coupler using cuprous oxide. The coupling efficiency, reflection coefficient, return loss and VSWR for different input signal wavelengths have been determined and presented. Our proposed coupler yields a coupling efficiency of $56 \%$ at $1,550 \mathrm{~nm}$ wavelength and the impedance mismatch is also quite small. We expect that the analysis presented here will be useful in the fabrication process of nanoplasmonic couplers.

Acknowledgments The author would like to acknowledge the support of Islamic University of Technology (IUT), Bangladesh for providing the resources.

Open Access This article is distributed under the terms of the Creative Commons Attribution License which permits any use, distribution, and reproduction in any medium, provided the original author(s) and the source are credited.

\section{References}

Al-Jabr A, Alsunaidi M (2009) An efficient time-domain algorithm for the simulation of heterogeneous dispersive structures. J Infrared Millim Terahertz Waves 30(11):1226-1233

Alsunaidi MA, Al-Jabr AA (2009) A general ADE-FDTD algorithm for the simulation of dispersive structures. Photon Technol Lett IEEE 21(12):817-819

Barnes WL, Dereux A, Ebbesen TW (2003) Surface plasmon subwavelength optics. Nature 424(6950):824-830

Berenger JP (1994) A perfectly matched layer for the absorption of electromagnetic waves. J Comput Phys 114(2):185-200

Ginzburg P, Orenstein M (2007) Plasmonic transmission lines: from micro to nano scale with $\lambda / 4$ impedance matching. Opt Express 15(11):6762-6767 
Kunz KS, Luebbers RJ (1993) The finite difference time domain method for electromagnetics. CRC Press, Boca Raton

Palik ED (1998) Handbook of optical constants of solids, vol 3. Academic Press, New York

Pile D, Gramotnev DK (2006) Adiabatic and nonadiabatic nanofocusing of plasmons by tapered gap plasmon waveguides. Appl Phys Lett 89(4):041111-041113

Rakic AD, Djurišic AB, Elazar JM, Majewski ML (1998) Optical properties of metallic films for vertical-cavity optoelectronic devices. Appl Opt 37(22):5271-5283

Taflove A, Hagness SC (2000) Computational electrodynamics, vol 160. Artech house, Boston

Veronis G, Fan S (2007) Theoretical investigation of compact couplers between dielectric slab waveguides and two- dimensional metal-dielectric-metal plasmonic waveguides. Opt Express 15(3):1211-1221

Wahsheh R, Lu Z, Abushagur M (2012) Nanoplasmonic air-slot coupler: design and fabrication. In: Frontiers in optics 2012. Rochester, New York, USA. doi:10.1364/FIO.2012.FTh4A.6

Yee K (1966) Numerical solution of initial boundary value problems involving Maxwell's equations in isotropic media. IEEE Trans Antennas Propag 14(3):302-307

Zia R, Selker MD, Catrysse PB, Brongersma ML (2004) Geometries and materials for subwavelength surface plasmon modes. JOSA A 21(12):2442-2446 